\title{
La qualité des systèmes d'enseignement des sciences et des techniques à l'aune de fondements épistémologiques des sciences cognitives
}

\author{
Mohamed BAHRA ${ }^{1}$ \\ Aouatif NAJOUA ${ }^{2}$, Fatima ABBASSI ${ }^{3}$ \\ Achouaq AAZIM ${ }^{4}$, Hafida BOUANANI ${ }^{5}$.
}

\author{
${ }^{1}$ Centre Régional des métiers de la Formation et de l'Education, Casablanca. \\ ${ }^{2}$ ENSET, Université Hassan II, Mohammedia/Casablanca \\ ${ }^{3}$ ENSET, Université Hassan II, Mohammedia/Casablanca \\ ${ }^{4}$ ENSET, Université Mohamed V, RABAT-Souissi \\ ${ }^{5}$ Centre Régional des métiers de la Formation et de l'Education, Safi
}

\section{Résumé :}

Le tableau noir est l'archétype des médias qui accueillent difficilement l'interactivité. Or, bien que la cybernétique se soit imposée, depuis les années 40 du siècle dernier, comme nouveau paradigme quant à la conceptualisation des activités cognitives et intellectuelles, les pratiques scolaires restent attachées au tableau noir comme principal médium dans la transmission des savoirs. Et bien qu'on ait passé, à l'aube de ce siècle, de la cybernétique à la cognitique, le tableau noir, devenu blanc, conserve encore aujourd'hui sa place de médium quasi incontournable.

La prégnance du tableau noir est telle qu'il est difficile d'imaginer que la nature des contenus d'enseignement visés puisse déterminer les médiums qui leur sont idoines et que ceux-ci puissent être incompatibles avec le tableau noir. Quand le contenu comporte du dynamique et du statique le recours à ce tableau rend implicite le premier et ne laisse explicite que le second. Les manipulations, en travaux pratiques, n'échappent pas à l'emprise de cet abord didactique statique immanent au tableau noir : ces manipulations se réduisent le plus souvent à de simples monstrations. Nous tentons de montrer en quoi il y a un modelage très robuste, bien qu'imperceptible de la part de la noosphère, de la structuration des contenus d'enseignement selon un moule unique, celui dicté par le tableau noir et que ce moule vide ces contenus de leur teneur morpho-dynamique. La qualité d'enseignement est hypothéquée par ce moule.

\section{Mots clés :}

Segmentation thématique - représentation scénique - praxéologie - sériation chronologique modélisation - sténographie cognitive 


\section{Première partie}

Argumentaire du symposium : Une problématique, un objet de recherche et une méthode d'approche relatives à la qualité des systèmes d'enseignement des sciences et des techniques

I. Problématique : Schème morpho-dynamique et représentation numérisée des connaissances en sciences et techniques ou stipulations du franchissement d'un obstacle didactique insoupçonné: 'le tableau noir'.

Depuis les années 40 du siècle dernier, la cybernétique s'est imposée comme nouveau paradigme quant à la conceptualisation des activités cognitives. À l'aube du siècle courant, via la cognitique, elle se réimpose dans le domaine de la conceptualisation de la communication et de l'interaction sémiotique. Cette évolution dans les domaines de la communication et de la cognition n'a pas encore d'effets significatifs sur les pratiques scolaires qui continuent d'utiliser le traditionnel tableau noir comme principal support pour sténographier des connaissances.

En effet, tenir compte de cette évolution veut que cette sténographie puisse produire des sténogrammes capables d'interaction sémiotique. Or, de par sa nature même, le tableau noir est l'archétype des supports qui accueillent difficilement de l'interactivité.

La prégnance du tableau noir serait d'ailleurs telle qu'il est difficile pour les promoteurs de ces pratiques scolaires d'imaginer que la nature des contenus d'enseignement puisse déterminer les médiums qui leur sont idoines et que ces derniers puissent être incompatibles avec ce support. A quoi donc cette incompatibilité serait-elle due quant aux savoirs visés par les systèmes d'enseignement des sciences et des techniques?

Ces savoirs sont des systèmes métacognitifs et la métacognition dont ils sont les vecteurs devrait être basée sur une sténographie des connaissances qui, en tant que telle, devrait être pourvoyeuse de sténogrammes capables d'interaction sémiotique. Aussi, d'un niveau d'enseignement à un autre, chacun de ces savoirs ne pourrait être appréhendé par l'élève que comme émergent d'une sténographie évolutive qui aurait pour objet le redéploiement progressif d'une conceptualisation bien établie d'un domaine de connaissances assez circonscrit.

La genèse de cette sténographie cognitive, par laquelle ce redéploiement advient, exigerait la coprésence d'un système relationnel empirique et d'un système relationnel numérique homomorphes et répondant aux deux modalités suivantes :

1. le premier système s'articule autour de la donnée de deux entités complémentaires :

a. un ensemble d'objets concrets susceptibles de former des groupements de modules, chaque groupement permettant la composition d'une configuration d'objets. L'ensemble est à l'image des pièces mélangées d'une famille de puzzles.

b. un système opératoire qui, opérant sur ces objets concrets, permet de réaliser chaque fois, quand c'est possible, la configuration correspondante (voire le 
puzzle correspondant) à un groupe d'objets choisis arbitrairement ou intentionnellement par un sujet actant.

2. le second système s'articule autour d'un alphabet L de lettres, à l'image des notations conventionnelles d'un champ formulaire, et d'une partition de l'ensemble $L^{*}$ des mots (formules) écrits(es) sur L en les trois classes suivantes :

a. la première classe est la classe des mots qui rendent compte de quelque relation vérifiable dans le premier système, comme rendre compte de la possibilité, imperceptible auparavant, de la formation, via le système opératoire, d'une configuration en particulier. Cette classe rassemble les mots de $\mathrm{L}^{*}$ qui jouissent, en tant qu'énoncés, de la double validité, syntaxique et sémantique : des mots de $\mathrm{L}^{*}$ qui signifient.

b. la seconde classe rassemble les mots qui peuvent rendre compte de quelque relation vérifiable dans le premier système, sous la condition de procéder à une métamorphose du système opératoire qui aurait pour aboutissement l'extension de son domaine de validité. L'adjonction de nouveaux objets concrets aux objets anciens du système empirique peut s'avérer elle aussi, à cette occasion, nécessaire. Les configurations à composer peuvent aussi voir leur liste s'allonger.

Cette seconde classe rassemble les mots de L* qui jouissent, en tant qu'énoncés, de la validité syntaxique et qui voient leur validité sémantique suspendue à une évolution possible, telle celle décrite ci-dessus, dans l'organisation du système relationnel empirique.

c. la troisième classe est constituée des mots qui ne peuvent rendre compte d'aucune relation vérifiable dans le système relationnel empirique quels que soient les changements que l'on puisse apporter à ce système.

Cette troisième classe rassemble les mots de $L^{*}$ qui ne peuvent jouir, en tant qu'énoncés, de la validité syntaxique.

Ainsi est-il loisible de qualifier d' « effectivement corrects » les mots de la première classe, de « virtuellement corrects » les mots de la seconde et d' « absolument incorrects » les mots de la troisième et considérer que chaque mot effectivement correct, pris avec son renvoi à la configuration d'objets dont il rend compte, est le sténogramme d'une connaissance relative au savoir enjeu de l'activité sténographique.

Proprement dit, le savoir enjeu de l'activité sténographique peut être conçu comme étant le compte rendu complet des métamorphoses successives que l'on fait subir au système relationnel empirique afin, chaque fois, de procéder à une extension du domaine de validité de son système opératoire et ce, afin de réintégrer dans la classe des mots effectivement corrects une famille donnée de mots virtuellement corrects.

Or, les métamorphoses successives du système relationnel empirique font que des configurations distincts d'objets, et donc auxquelles renvoient des mots corrects distincts, peuvent être déclarées configurations équivalentes; aussi, les mots corrects qui rendent compte de ces configurations doivent alors être considérés comme autant de sténogrammes d'une même et seule connaissance. Ainsi, des sténogrammes distincts peuvent représenter une seule et unique connaissance. Mais, le savoir, en tant qu'il est le compte rendu complet de ces métamorphoses, leurs tenants et leurs aboutissants, admet une fonction synthétisante. Celle-ci 
identifie chacune des connaissances relatives au savoir par de-là la diversité de ses représentations sténographiques, sans ambiguïté ni redondance aucunes.

Cette fonction synthétisante du savoir suggère distinguer deux types d'approches didactiques pour un savoir donné :

1. La première approche est aveugle à l'équivalence de configurations et consiste à énumérer les connaissances relatives au savoir en adoptant comme principe de discrimination entre ces connaissances celui de la discrimination des sténogrammes ou des mots effectivement corrects. Les connaissances sont alors prises pour elles-mêmes sans qu'il soit question de les rapporter à ce savoir auquel elles sont pourtant reliées : deux sténogrammes distincts d'une même et seule connaissance relative au savoir en jeu étant traités dans ce type d'approche comme s'il s'agissait de sténogrammes de connaissances distinctes. Avec ce type d'approches il est impossible pour les pratiques didactiques de faire fonctionner dans leur fonction synthétisante les savoirs qu'elles visent.

2. La seconde approche tient compte de l'équivalence de configurations et la rapporte à la dynamique des intégrations successives de familles de mots virtuellement corrects dans la classe des mots effectivement corrects via des métamorphoses successives du système relationnel empirique, notamment par l'extension du domaine de validité de son système opératoire à ces familles de mots virtuellement corrects. Dans cette approche, les connaissances sont rapportées au savoir auquel elles sont reliées puisque ce savoir, étant le compte rendu complet de ces métamorphoses et extensions, ainsi que de leurs motivations, est, entre autres, énumération, pour chacune des connaissances, de tous les sténogrammes y afférents. Ainsi, ce serait seulement avec ce type d'approches qu'il devient possible de faire fonctionner le savoir visé dans sa fonction synthétisante.

Il est donc loisible de déclarer non synthétisantes, quant aux connaissances relatives au savoir qu'elles visent, les approches didactiques de premier type et synthétisantes, les approches de second type. Insistons sur le fait que ces dernières sont caractérisées par leur capacité à distinguer chacune des connaissances en jeu par-delà la multiplicité de ses représentations sténographiques. C'est la capacité à confondre les connaissances associées à des configurations qui, bien que distinctes, sont équivalentes, ces équivalences dérivant de manière nécessaire de la succession des métamorphoses du système relationnel empirique tendant à étendre la validité sémantique à tous les énoncés qui jouissent de la validité syntaxique.

Qu'un sujet actant puisse assister au déploiement de la fonction synthétisante d'un savoir qui, rappelons-le, consiste à confondre les connaissances dont les sténogrammes renvoient à des configurations équivalentes d'objet dans le système relationnel empirique, cela nécessite rendre accessible pour cet observateur l'historique des configurations, des sténogrammes et des métamorphoses du système relationnel empirique. Ces sténogrammes doivent aussi être capables d'interaction sémiotique de telle manière que l'observateur puisse reconnaître, en naviguant dans cet historique, l'accumulation des configurations équivalentes et les sténogrammes les dénotant dans la succession des métamorphoses du système relationnel empirique. 
Pour rendre disponible une telle navigation, le support de l'historique doit pouvoir permettre la mobilisation simultanée d'une multitude de ses propres occurrences à l'image d'une multitude d'écrans superposés, offrant un nombre suffisamment grand de possibilités de parcours. Le tableau noir ne pourrait être ce support. De fait, le recours des pratiques scolaires au tableau noir traditionnel se constituerait en obstacle à l'émergence et à la promotion dans le milieu scolaire des approches didactiques synthétisantes au profit de la promotion d'approches didactiques non synthétisantes.

C'est ainsi que si, pour qu'il puisse garantir des enseignements de qualité, un système d'enseignement des sciences et des techniques se doit d'être doté d'un mécanisme de régulation lui permettant de détecter puis corriger, à temps, toute tendance du système à promouvoir des approches didactiques non synthétisantes et ce, par la substitution, à cette tendance, la tendance à promouvoir des approches didactiques synthétisantes, alors la numérisation des contenus d'enseignement et leur adaptation à la navigation susmentionnée et ses supports devient de plus en plus une nécessité vitale.

Notons que l'emprise, via l'usage du tableau noir, des approches didactiques non synthétisantes sur les pratiques scolaires et l'éviction, par celles-ci, des approches didactiques synthétisantes n'épargne pas celles parmi ces pratiques qui visent faire fonctionner un savoir via un dispositif expérimental sensé permettre le déploiement de sa fonction synthétisante. En effet, tout montage expérimental motivé doit être le résultat d'un travail de conceptualisation de ce même déploiement, travail auquel l'élève doit participer activement ; aussi, ce travail ne saurait se réaliser que dans le cadre d'une approche didactique synthétisante. Cet type d'approche étant quasiment interdit par le recours des pratiques scolaires au tableau noir ou assimilés, ces dernières sont acculées à opérer une réduction du processus expérimental en une monstration non informée des véritables motifs et ressorts de l'activité sous-jacente.

Ainsi, tout se passe comme si les pratiques didactiques fonctionnent selon l'hypothèse erronée suivante: toute approche didactique non synthétisante est porteuse des germes d'une approche didactique synthétisante. Nous entendons par là qu'à mesure que les pratiques scolaires, en tant que destinatrice, avancent dans le travail qui consiste à énumérer, à l'adresse de l'élève, en tant que destinataire, les connaissances relatives à un savoir donné, tout en faisant, éventuellement, fonctionner ces connaissances indépendamment les unes des autres, ces connaissances finissent par se regrouper spontanément chez le destinataire en des groupes de connaissances identiques car se rapportant à des configurations équivalentes.

Pour donner une idée de la portée du caractère erroné de cette hypothèse, nous proposons de le dénommer «hypothèse du schème morpho-dynamique spontané ».

Une des préoccupations majeures de la didactique des sciences et des techniques devrait être de montrer, via un usage spécifique des TIC, que, sous l'emprise du tableau noir comme médium privilégié, les pratiques scolaires fonctionnaient bel et bien sous l'hypothèse du schème morpho-dynamique spontané et que cette hypothèse est une hypothèse erronée. 


\section{Objet de recherche et méthode d'approche : Les quatre stipulations d'un recadrage nécessaire d'objets d'apprentissage relatifs à des objets d'enseignement des sciences et des techniques}

Pour montrer que les pratiques scolaires fonctionnent sous «l'hypothèse du schème morphodynamique spontané » et que cette hypothèse est erronée, nous estimons convenable de montrer que le déploiement du schème morpho-dynamique chez l'élève ne saurait avoir lieu sans l'actualisation de morphogenèses spécifiques comme substratum du schème. Partant du fait que cette actualisation est difficile à obtenir sur un support tel que le tableau noir, l'on s'attend à ce que la forme prise par les objets d'apprentissage soient trop éloignée de la forme adéquate jusqu'à se constituer en obstacle à la formation de conceptions valides chez l'élève.

Aussi, la spécification de cet obstacle et la recherche des conditions générales organisant son franchissement, nécessiteraient-elles d'opposer à des objets d'apprentissages, tels qu'ils sont institutionnalisés par les pratiques didactiques courantes, des objets d'apprentissages, portant sur les mêmes objets d'enseignement mais répondant aux stipulations de l'interaction sémiotique selon des techniques que nous dérivons de fondements épistémologiques des sciences cognitives. La question essentielle est de savoir lequel des deux types d'objets d'apprentissage est l'interprète fidèle des objets d'enseignements visés.

Les objets d'apprentissage dont il s'agira ici sont ceux à travers lesquels les pratiques didactiques traduisent les objets officiels d'enseignement portant sur :

1. les modèles mathématiques de mouvements rectilignes, du mouvement des corps en chute libre, dont le mouvement du projectile ;

2. les dispositifs expérimentaux visant l'établissement de la conformité de ces modèles mathématiques à la réalité ;

3. les concepts de physique permettant d'avancer l'explication du pourquoi de la conformité de ces modèles mathématiques à la réalité.

Les techniques que nous dérivons de fondements épistémologiques des sciences cognitives et qui nous servirons de grille de lecture des objets d'apprentissage et des objets d'enseignement considérés concerneront la structuration du discours enseignant, des contenus d'enseignement à teneur praxéologique et des interventions didactiques en les sciences et les techniques, ainsi que la constitution des objets d'apprentissages comme projection cartographique des objets d'enseignement correspondants. Voici les linéaments de chacun de ces quatre axes.

\section{A. La structuration du discours de l'enseignant}

Il s'agit de la structuration du discours de l'enseigna nt dans ce que cette structuration a de spécifique :

- aux segmentations thématiques que le discours opère sur l'ensemble des objets concrets du système relationnel empirique et sur l'ensemble des mots du système relationnel numérique; il s'agit :

- de deux segmentations simultanées de premier degré : 
- dans le premier ensemble, sous le thème «configuration bien formée », la première segmentation tend à l'explicitation des groupes de modules donnant chacun une configuration bien formée d'objets ;

- dans le second ensemble, sous le thème "sténogramme effectif ou potentiel de connaissance », la seconde segmentation, simultanée à la première, tend à l'explicitation de la classe des mots effectivement corrects, celle des mots potentiellement corrects et celle des mots absolument incorrects.

○ d'une segmentation de second degré consistant à regrouper les configurations équivalentes et les sténogrammes qui leur correspondent en rapportant ces équivalences à une métamorphoses du système opératoire, outil de production de configurations bien formées, métamorphose dictée par l'intégration, devenue quasi nécessaire, d'une famille spécifiée de mots virtuellement corrects dans la classe des mots effectivement corrects.

- à la régulation de représentations scéniques chez l'apprenant ; il s'agit :

- d'une représentation scénique de premier degré consistant à se représenter les mises en branle du système opératoire suite à la formation, accompagnant les segmentations thématiques du premier degré, de mots effectivement ou potentiellement corrects et de configurations d'objets appartenant au substrat du système relationnel empirique ;

- d'une représentation scénique de second degré consistant à se représenter les métamorphoses successives subies par le système opératoire; chaque métamorphose est dictée par l'intégration, devenue quasi nécessaire, d'une nouvelle famille spécifiée de mots potentiellement corrects dans la classe des mots effectivement corrects.

Notons que la segmentation thématique de second degré et la représentation scénique de second degré sont nécessaires pour que le discours puisse faire fonctionner le savoir, par lui visé, dans sa fonction synthétisante.

\section{B. la structuration des contenus d'enseignement dans ce que ces contenus ont de spécifique à la teneur praxéologique des savoirs visés.}

Ces savoirs, étant considérés alors comme autant de missions en attente de réaccomplissement par l'apprenant, celles-ci devraient suggérer les micro-missions et les mésomissions dont la réalisation est nécessaire pour ce ré-accomplissement.

Un système opératoire s'érige au niveau d'une praxéologie quand le système est considéré, non pas pour ce qu'il est à travers des occurrences de sa mise en application, mais pour la possibilité de le voir débordé par des mots potentiellement corrects et qu'il faille concevoir sa métamorphose pour contenir ce débordement, notamment en faisant évoluer le statut de ces mots de potentiellement corrects à effectivement corrects.

Pour souligner le lien entre ce point de vue et les contenus d'enseignement à teneur praxéologique nous avançons les assertions suivantes : 
- En enseignement des sciences et des techniques un savoir, en tant qu'il est une œuvre, est une mission qui consiste en la capture sténographique des connaissances relatives à l'intelligibilité et/ou la maîtrise de quelque phénoménologie. Dit plus succinctement, un savoir scientifique et/ou technique, dans le contexte de l'enseignement et de l'apprentissage, est une mission qui consiste en la capture sténographique de quelque phénoménologie.

- D'un point de vue praxéologique, cette capture suppose l'existence de pratiques auxquelles on se livrait autour de la phénoménologie visée. Aussi fait-on correspondre à une famille de pratiques, que l'on peut considérer comme élémentaires, une famille de pratiques de second degré : une combinaison de pratiques issues de la première famille et qui se prêtent à un usage parallèle donne lieu, via cet usage, à une nouvelle pratique de niveau supérieur que nous dénommons pratique de second degré. Nous supposons l'existence d'un seuil dans le nombre de pratiques de second degré en deçà duquel la maîtrise de ces pratiques reste insuffisante pour que la capture sténographique complète de la phénoménologie visée ait lieu.

- Les pratiques élémentaires sont aux pratiques de second degré ce que sont les lettres de l'alphabet L aux mots écrits sur L, suites finies d'éléments de L et sténogrammes potentiels. Les pratiques de second degré sont à la capture sténographique ce que sont les mots corrects initiaux aux vagues successives de familles de mots qui, successivement, ont vu leur statut changer de potentiellement corrects à effectivement corrects.

\section{La structuration des interventions didactiques, dans ce que cette structuration a de spécifique aux possibilités, tant théoriques que pratiques, de rendre les systèmes informatiques, du genre TBI et tablettes, des systèmes capables d'interaction sémiotiques.}

Le principal apport des systèmes informatiques, tels que TBI et Tablettes, est l'opportunité que ces systèmes offrent de faire de cette interaction une composante essentielle du canal quand il s'agit de la communication d'un message dont le contenu est un savoir scientifique et/ou technique spécifié.

Pour saisir cette opportunité, il serait nécessaire de baser la structuration de l'intervention didactique sur quatre niveaux d'articulation :

- le niveau morphologique où il faut déterminer les systèmes relationnels, empirique et numérique, idoines pour la constitution du substrat de la composante, dédiée à l'interaction sémiotique, du canal d'où faire transiter, à l'adresse de l'élève, le message dont le contenu est le savoir visé par l'intervention didactique.

- Le niveau syntaxique où il faut déterminer les conditions objectives sous lesquelles l'élève pourra tenir le rôle de l'agent épistémique formant des mots et cherchant à connaître le statut de chaque mot formé eu égard au fait que le mot spécifie une liste d'objets et rend compte soit de la possibilité effective, soit de la 
plausibilité virtuelle, soit de l'impossibilité absolue de constituer avec ces objets une configuration bien formée: le mot alors sera qualifié, selon le cas, d'effectivement correct, de potentiellement correct ou d'absolument incorrect.

- Le niveau sémantique où il faut déterminer les conditions objectives sous lesquelles l'élève pourra tenir le rôle de l'agent épistémique cherchant à procéder à des extensions successives du domaine de validité du système opératoire de façon à pouvoir chaque fois réintégrer une famille de mots potentiellement corrects dans la classe des mots effectivement corrects.

- Le niveau pragmatique où il faut déterminer les conditions objectives sous lesquelles l'élève pourra tenir le rôle de l'agent épistémique cherchant à continuer le processus des extensions successives du domaine de validité du système opératoire jusqu'à l'obtention du système au domaine de validité le plus vaste, celui-ci tendant à réintégrer la totalité des mots potentiellement correct dans la classe des mots effectivement corrects.

Les deux premiers niveaux préparent à l'établissement par l'élève des connaissances relatives au savoir visé ; tandis que les deux derniers niveaux préparent à le mettre dans les conditions qui lui permettraient de tirer toutes les conséquences logiques de ces connaissances. Cela suggère, pour les interventions didactiques, de confondre tout savoir avec les conséquences logiques de connaissances spécifiques, celles relatives à ce savoir. Une autre façon de dire que les extensions successives du domaine de validité du système opératoire constitutif du système relationnel empirique s'appréhendent comme les conséquences logiques de la formation des trois classes des mots et c'est en tirant ces conséquences qu'on établit le compte rendu complet de ces extensions, soit le savoir visé au travers la constitution des deux systèmes homomorphe, l'empirique et le numérique.

\section{Les objets d'apprentissage comme résultats d'une projection de type cartographique appliquée par les pratiques didactiques aux objets d'enseignement qu'elles visent.}

Avec l'avènement de ces systèmes informatiques que l'ingénierie didactique peut transformer en une composante interactive du canal assurant la transmission, à l'adresse de l'apprenant, de messages dont les contenus sont des savoirs déterminés, l'objet d'apprentissage gagnerait à être décrit comme le résultat d'une projection cartographique de l'objet d'enseignement correspondant et ce, pour les raisons qui suivent :

- les termes d'une modélisation formelle (mathématique) d'une phénoménologie dont on cherche la maîtrise et l'intelligibilité peuvent correspondre à des signifiants dans le langage usuel ou dans le langage de la modélisation. Malgré le fait que les signifiés correspondants soient des entités abstraites, le simple fait que ces termes soient des signifiants langagiers donne l'impression que cette modélisation formelle se suffit à elle-même pour assurer sa propre validité sémantique. Ainsi, la maîtrise des aspects formels de la modélisation, la possibilité d'effectuer des calculs sur 
ces aspects, va donner l'impression que la phénoménologie modélisée est devenue intelligible alors qu'il n'en est, peut-être, rien. En fait, le modèle va prendre une des deux formes suivantes :

- soit comme une structure qui, bien qu'ouverte à une diversité d'actualisations, est conçue comme se suffisant à elle-même et, en tant que telle, n'a pas besoin d'être accompagnée d'une explicitation, suffisamment assumée, d'une de ses actualisations ;

- soit comme une explication causale des faits d'observations, relatifs à la phénoménologie, mais basée sur une modélisation formelle implicite.

Chacune de ces deux formes rappelle une projection cartographique, sur le plan, d'une structure complexe tridimensionnelle: Des points fondamentaux de la structure sont représentés dans la forme projetée par des points cachés sous des points du plan représentant des points sans grande importance dans la structure originelle. En vertu de quoi, pour admettre l'adéquation de la projection cartographique choisie quant à la structure d'origine, il faudrait pouvoir trouver assez facilement dans la forme projetée des traces suffisamment prégnantes de l'état sous lequel le savoir visé apparaît dans les monographies universitaires les plus avancées. Nous entendons par projection cartographique l'objet d'apprentissage tel qu'il se présente dans les manuels scolaires ou dans une ingénierie didactique spécifique et par «savoir visé »celui visé par l'objet d'enseignement en tant que cet objet est une synthèse des états de ce savoir, et donc de la structure d'origine, dont ce même objet d'apprentissage est une des traductions possibles.

- Les concepts de nombre et de grandeur occupent une place prépondérante dans la modélisation formelle de phénoménologies. En construisant le contenu de ses messages sur ces deux concepts, l'émetteur (qui n'est autre que la noosphère puisque, dans les faits, le professeur fait partie du canal) fait comme si cela est suffisant pour que les messages puissent permettre au récepteur d'accéder à la maîtrise et à l'intelligibilité de la phénoménologie. En fait, ces messages ne sauraient conduire le récepteur à l'intelligibilité et la maîtrise de la phénoménologie sans que soit rejetée sur cette dernière une morphologie sténographique de type géométrique censée avoir cette même phénoménologie comme une de ses propres actualisations à une échelle plus ou moins grande. Encore faut-il que l'émetteur admette que cette actualisation ne puisse être appréhendée que par le récepteur tenant le rôle de l'agent épistémique qui se pose le problème de la conformité de cette traduction géométrique à la réalité phénoménologique et essaye de le résoudre. Insistons sur le fait que c'est dans le cadre de la conception et réalisation de dispositifs expérimentaux au sein de laboratoires scientifiques et d'ateliers technologiques que la résolution de ce problème pourrait être entreprise. Notons aussi à ce propos que, très souvent, la simulation 
informatisée des expériences de la physique est utilisée malencontreusement comme pour éviter de faire tenir au récepteur le rôle de l'agent épistémique susmentionné. Quand c'est le cas, la simulation donne l'impression de donner lieu à de l'interaction sémiotique alors qu'il n'en est absolument rien... 


\section{Deuxième partie}

\section{Les 4 axes de symposiums à l'œuvre dans une mise à l'épreuve du rapport}

des systèmes d'enseignement des sciences et des techniques à l'objet de savoir 'triangle rectangle'

\section{Trois segmentations thématiques autour du triangle rectangle.}

\section{A. Notion intuitive de segmentation thématique}

Intuitivement, une segmentation thématique renvoie sans s'y identifier complétement à un système à états évolutif, de sorte que :

- chaque état du système est une occurrence du thème conducteur de la segmentation ;

- l'historique des états du système est la trajectoire du thème ;

- un espace formé de trajectoires de thèmes analogues peut être désigné référentiel du thème ;

- est frontière du thème toute délimitation dans le référentiel d'un intérieur et d'un extérieur topologiques, l'intérieur contenant la trajectoire du thème.

Opérer une segmentation thématique sur quelque substrat consiste à y révéler progressivement les occurrences d'un thème, la trajectoire de ce thème, son référentiel et sa frontière.

Selon cette présentation intuitive de cette action, il est possible d'opérer sur le triangle rectangle au moins trois segmentations thématiques dont on peut dire qu'ils sont spécifiques à cette forme géométrique.

Ainsi, à propos d'un triangle XOY rectangle en $\mathrm{O}$, l'on peut envisager les segmentations thématiques suivantes :

1. Segmentation thématique relative aux aires de trapèzes contenus dans le triangle et dont [OY] est une des deux bases.

Dotant le plan d'un repère orthonormé $(\mathrm{O} ; \mathrm{I} ; \mathrm{J})$ avec I un point de $[\mathrm{OX})$ et $\mathrm{J}$, un point de [OY).

A toute subdivision du côté $[O X]$ en $n$ intervalles $\left[T_{i-1} T_{i}\right]$ avec $i=0,1, \ldots, n, T_{i}$ entre $T_{i-1}$ et $\mathrm{T}_{\mathrm{i}+1}$, pour $\mathrm{i}=1, \ldots, \mathrm{n}-1, \quad \mathrm{~T}_{0}=\mathrm{O}$ et $\mathrm{T}_{\mathrm{n}}=\mathrm{X}$, fait apparaître les points $\mathrm{Y}_{\mathrm{i}}$ d'abscisse 0 et d'ordonnée le nombre mesure de l'aire du trapèze $O Y R_{i} T_{i}$, avec $R_{i}$ le point où la parallèle à $(\mathrm{OY})$ issue de $\mathrm{T}_{\mathrm{i}}$ coupe [YX], comme les marques d'une segmentation thématique opérée sur l'axe $(\mathrm{O} ; \mathrm{J})$, via la correspondance qui associe à chaque rang $\mathrm{i}$, les points $\mathrm{T}_{\mathrm{i}}$ et $\mathrm{Y}_{\mathrm{i}}$ :

- pour tout rang $\mathrm{i}$, le point $\mathrm{Y}_{\mathrm{i}}$ est une occurrence du thème conducteur de la segmentation ;

- la trajectoire de ce thème est l'intervalle [OY $], Y_{n}$ étant le point d'abscisse 0 et d'ordonnée le nombre mesure de l'aire du triangle OYX ;

- le référentiel du thème est la demi-droite [OY) ;

- $\quad$ et sa frontière, les points $\mathrm{O}$ et $\mathrm{Y}_{\mathrm{n}}$.

Cette segmentation thématique originelle se complexifie par les deux autres suivantes :

2. Segmentation thématique relative aux taux de variation des aires de polygones prolongeant les trapèzes $O \mathbf{Y R}_{\mathbf{i}} \mathbf{T}_{\mathbf{i}}$.

Pour tout rang i, avec i allant de 0 à n, on pose : 
- $\quad T_{n+i}$ le symétrique par rapport à $T_{n}$ de $T_{n-i} ;$

- $\quad \mathrm{R}_{\mathrm{n}+\mathrm{i}}$, l'intersection de la parallèle à (OY) issue de $\mathrm{T}_{\mathrm{n}+\mathrm{i}}$ avec (XY) ;

- et, pour tout rang $\mathrm{j}$ compris entre $\mathrm{i}$ et $2 \mathrm{n}, \mathrm{R}{ }_{\mathrm{ij}}$, le point intersection de la parallèle à $(\mathrm{OY})$ issue de $T_{\mathrm{j}}$ avec la parallèle à $(\mathrm{OX})$ issue de $\mathrm{R}_{\mathrm{i}}$.

$\mathrm{Si}$ on décide de remplacer pour tout rang $\mathrm{j}$ supérieur ou égal à $\mathrm{i}$ le point $\mathrm{R}_{\mathrm{j}}$ par le point $\mathrm{R}_{\mathrm{ij}}$, l'on considère alors les polygones $O Y R_{i} R^{\prime}{ }_{i j} T_{j}$. En passant du rang $j$ au rang $j+1$ l'aire $d u$ polygone OYRiR' ${ }_{i j} T_{j}$ subit une augmentation de $T_{j} T_{j+1} X T_{j} R^{\prime}{ }_{i j}$ égale à $T_{j} T_{j+1} X T_{i} R_{i}$. Une augmentation proportionnelle au pas $T_{j} T_{j+1}$ et dont le coefficient de proportionnalité est $T_{i} R_{i}$. La considération pour chaque rang $i$ des polygones $O Y R_{i} R^{\prime}{ }_{i j} T_{j}$, avec $j$ variant entre $i$ et $2 n$, fait apparaître les segments $\left[\mathrm{T}_{\mathrm{i}} \mathrm{R}_{\mathrm{i}}\right]$, pour $\mathrm{i}$ allant de 0 à $\mathrm{n},\left[\mathrm{T}_{\mathrm{i}} \mathrm{R}_{\mathrm{i}}\right]$ étant pris avec son renvoi au coefficient de proportionnalité $\mathrm{T}_{\mathrm{i}} \mathrm{R}_{\mathrm{i}}$, comme les marques d'une segmentation thématique opérée sur le triangle OYX et dont :

- chaque segment $\left[\mathrm{T}_{\mathrm{i}} \mathrm{R}_{\mathrm{i}}\right]$ est une occurrence du thème conducteur de la segmentation ;

- $\quad$ le segment [XY], la trajectoire du thème ;

- le triangle OYX représente le référentiel du thème (espace couvert les segments $[\mathrm{TR}])$;

le produit des deux côtés $[\mathrm{OX}]$ et $[\mathrm{YX}]$, constitue la frontière du thème.

\section{Segmentation thématique relative au taux de variation des taux de variations $\mathbf{T}_{\mathbf{i}} \mathbf{R}_{\mathbf{i}}$.}

\section{a. Eléments introductifs}

$\mathrm{T}$ est un point de $[\mathrm{OX}]$, RT et $\mathrm{ST}$, respectivement intersection de $(\mathrm{dT})$, la perpendiculaire à $(\mathrm{OX})$ issue de $\mathrm{T}$, avec, respectivement, $[\mathrm{XY}]$ et $\left(\mathrm{D}^{\prime}\right)$, la perpendiculaire à $(\mathrm{OY})$ issue de $\mathrm{Y}$. L'on a : TR $=$ OST - STR $=$ OY - STR. Les variations de TRT, quand T parcours $[O X]$ sont alors les variations de -STRT quand T parcours [OX]. Aussi, considère-t-on Y', le point tel que OYXY' soit un parallélogramme, R' T, l'intersection de (dT) avec [OY' ]; les variations de TR sont celles de -TR' 'T. En

outre, VT et V'T étant les projections orthogonales de R'' $\mathrm{T}$, respectivement sur (OY) et sur (D), la parallèle à (OY) passant par I, l'on considère le rectangle OVTV'TI. Le même nombre est mesure à la fois de la longueur du segment [TR' 'T] et de l'aire du rectangle OVTV'TI. Or, en considérant A' le point intersection de (OY' ') avec (D), la parallèle à (OX) passant par A' coupant (OY) et (TRT) respectivement en A et T', les rectangles OIVTV'T et OAT' $T$ ont même aire. Ainsi, le même nombre est à la fois mesure de la longueur du segment [TR' T] et de l'aire du rectangle OAT' $T$.

\section{b. La segmentation thématique relative aux longueurs $T_{i} R_{i}$}

Toute subdivision du côté $[\mathrm{OX}]$ en $\mathrm{n}$ intervalles $\left[\mathrm{T}_{\mathrm{i}-1} \mathrm{~T}_{\mathrm{i}}\right.$, avec $\mathrm{i}$ allant de 0 à $\mathrm{n}$, telle que $\mathrm{T} 0$ est confondu avec $\mathrm{O}$, Tn avec $\mathrm{X}$ et pour $\mathrm{i}$ allant de 1 à $n-1, T_{i}$ est entre $T_{i-1}$ et $T_{i+1}$, fait apparaître les points $\mathrm{VT}_{\mathrm{i}}$ comme les marques d'une segmentation thématique opérée sur l'axe $(\mathrm{O} ; \mathrm{J})$, via la correspondance qui associe à chaque rang i les point $\mathrm{T}_{\mathrm{i}}$ et $\mathrm{VT}_{\mathrm{i}}$ de sorte que :

- Pour tout rang $i$, le point $\mathrm{VT}_{\mathrm{i}}$ est une occurrence du thème conducteur de la segmentation ;

- Le segment $\left[\mathrm{O} \mathrm{VT}_{\mathrm{n}}\right]$ est la trajectoire du thème ;

- $\quad \mathrm{O}$ et $\mathrm{VT}_{\mathrm{n}}$ la frontière du thème ;

- $\quad[\mathrm{OA})$, le référentielle du thème. 


\section{c. Segmentation thématique relative au taux de variation des taux de variations des longueurs $\mathbf{T}_{\mathbf{i}} \mathbf{R}_{\mathbf{i}}$}

A Toute subdivision du côté $[\mathrm{OX}]$ en $n$ intervalles $\left[\mathrm{T}_{\mathrm{i}-1} \mathrm{~T}_{\mathrm{i}}\right]$, avec $\mathrm{i}$ allant de 0 à $\mathrm{n}$, telle que $\mathrm{T}_{0}$ est confondu avec $\mathrm{O}$, Tn avec $\mathrm{X}$ et, pour $\mathrm{i}$ allant de 1 à $n-1$, Ti est entre $\mathrm{T}_{\mathrm{i}-1}$ et $\mathrm{T}_{\mathrm{i}+1}$, faisons correspondre les $n$ rectangles $O A T{ }^{\prime}{ }_{i} T_{i}$. En passant du rang $i$ au rang $i+1$, l'aire du rectangle $O A T{ }^{\prime} T_{i}$ subit une augmentation égale à $\mathrm{OAxT}_{\mathrm{i}} \mathrm{T}_{\mathrm{i}+1}$. Cette augmentation est donc proportionnelle à $\mathrm{T}_{\mathrm{i}} \mathrm{T}_{\mathrm{i}+1}$ et le coefficient de proportionnalité et $\mathrm{OA}$.

Rappelons que les variations de TR sont celles de -TR''T. Aussi, le taux de variation des longueurs $\mathrm{T}_{\mathrm{i}} \mathrm{R}_{\mathrm{i}}$ est - OA l'ordonnée du point $\mathrm{A}$. Aussi, la segmentation thématique relative au taux de variation des taux de variations $\mathrm{T}_{\mathrm{i}} \mathrm{R}_{\mathrm{i}}$ se présente comme suit : le point $\mathrm{A}$ est à la fois thème, trajectoire, frontière du thème et la demi-droite d'origine $\mathrm{O}$, de support $(\mathrm{OJ})$ et ne passant pas par $\mathrm{J}$ est le référentiel du thème.

\section{A.B. Une représentation scénique, fusion interprétante des 3 segmentations thématiques du triangle rectangle}

\section{Une segmentation thématique de second degré issue des 3 segmentations du triangle rectangle}

L'apparence anodine des trois segmentations thématiques spécifiques au triangle rectangle est trompeuse. En effet, pour peu que l'on considère la demi-droite [OX), axe des temps, la demi-droite [OY), axe des positions de points mobiles animés de mouvements rectilignes, ces trois segmentations permettent de sélectionner un point parmi ces points en explicitant :

- le procédé qui permet la localisation de sa position Mt à tout instant $\mathrm{t}$;

- le procédé qui permet le calcul de la mesure algébrique $\overline{M_{t} M_{t+1}}$, lorsque, à partir de l'instant $\mathrm{t}$, le mouvement du point $\mathrm{M}$ est maintenu au moins pendant une durée couvrant une unité temps et que tout facteur faisant varier la vélocité avec laquelle le mobile passe de la position Mt à une position $\mathrm{Mt}+\alpha$, avec $0 \leq \alpha \leq 1$ est annihilé. L'on considère alors que $\overline{M_{t} M_{t+1}}$ est la vitesse du mobile à l'instant t, l'unité de la vitesse étant l'unité de la longueur OJ par unité de temps OI ;

le procédé qui permet de calculer la mesure algébrique $\overline{V_{t} V_{t+1}}$, lorsque, à partir de l'instant $\mathrm{t}$, le mouvement du point $\mathrm{V}$ est maintenu au moins pendant une durée couvrant une unité temps et que tout facteur faisant varier la vélocité avec laquelle le point $\mathrm{V}$ passe de la position $\mathrm{V}_{\mathrm{t}}$ à une position $\mathrm{V}_{\mathrm{t}+\alpha}$, avec $0 \leq \alpha \leq 1$ est annihilé. L'on considère alors que $\overline{V_{t} V_{t+1}}$ est l'accélération du point $\mathrm{M}$ à l'instant $\mathrm{t}$, l'unité de l'accélération étant l'unité de la longueur OJ par unité de temps OI élevée au carré: $:$;

\section{Questions fondant la représentation scénique interprétatrice des trois segmentations thématiques du triangle rectangle}

Ces trois procédés permettent de porter l'historicité kinesthésique du point $\mathrm{M}$, comme celle d'expériences effectives dans le milieu ambiant, à la formulation et à la description rationnelles. Cette formulation et cette description sont deux émergents de modèles mentaux de leur auteur, voire d'une représentation scénique du mouvement du point mobile M. La représentation scénique est l'activité mentale consistant à rejeter sur une segmentation thématique une sériation chronologique en vue de lui associer, comme une de ses conséquences logiques, un événement de nature cinesthésique (le mouvement de l'œil ou du 
doigt suivant un objet mobile). Les modèles de la cinématique du point en mathématiques sont des occurrences de telles segmentations se prolongeant en de telles représentations.

En effet, les trois procédés déroulés ci-dessus se prolongent naturellement en des questions non seulement formulables à l'adresse de l'élève encore assujetti à l'enseignement obligatoire, mais aussi à même de l'amener à rejeter sur les 3 segmentations thématiques du triangle une sériation chronologique des points de l'axe des ordonnées qui, très vite, peut se transformer en une représentation scénique d'un certain mouvement rectiligne uniformément varié.

Voici ces questions :

- En remarquant qu'avec le temps qui s'écoule, il y a simultanément augmentation de l'aire du trapèze $O Y R_{t} T$ et diminution de la longueur $T R_{t}$, à quoi le vecteur $T R_{t}$ pourrait-il renvoyer quand on sait que l'aire du trapèze renvoie à la position du point $\mathrm{M}$ à l'instant $\mathrm{t}$ ? justifier votre réponse par des réponses dûment argumentées aux questions suivantes :

$\ominus \quad$ En partant du point d'abscisse 0, comment s'effectue le départ de M, par comparaison à son arrivée en fin de course : brusquement, lentement ou également ?

$\ominus \quad$ Même question pour le point $\mathrm{V}$.

$\ominus \quad$ On peut dire du point M que son mouvement subit un effet de freinage. Pourquoi ? peut- on dire de l'intensité de ce freinage qu'elle est régulière ou irrégulière avec le temps ?

- Une phénoménologie courante et relativement simple pourrait être décrite, mais incomplètement, par le mouvement du point M. Quelle est cette phénoménologie et par quoi faut-il compléter le triangle OYX pour obtenir la description complète?

Une praxéologie spécifique, dans laquelle engager les élèves, adresse des questions ci-dessus, serait-elle un préalable pour que ces dernières puissent les placer au niveau de l'agent épistémique qui devant ces questions puissent donner des trois segmentations du triangle rectangle l'interprétation idoine ? Nous pensons à une praxéologie dont l'aboutissement serait une sténographie de perceptions kinesthésique.

\section{Praxéologie et sténographie cognitive : vers une sténographie de perceptions kinesthésiques externes.}

\section{A. Les tracés sur la feuille de papier érigés en une praxéologie cinématique via le système cartésien des cordonnées rectangulaires}

Une praxéologie est l'ensemble des stipulations tacites ou explicites qui s'imposent à un agent épistémique cherchant accomplir la mission qui consiste en la spécification de la conceptualisation d'un domaine de connaissances pratiques. Certainement, les trois segmentations thématiques du triangle rectangle et la représentation scénique qui en découlent, telles que présentées ci-dessus, s'inscrivent dans le cadre d'une praxéologie. Les objets d'enseignement impliqués dans ces segmentations thématiques et dans cette représentation scéniques sont censés être traductibles en objets d'apprentissage à même d'ériger l'élève au niveau de l'agent épistémique relatif à la praxéologie qui les subsume. Mais les objets d'enseignement ne sont pas accompagnés de moyens explicites de telles traductions. Ces moyens sont laissés à la discrétion des auteurs de manuels scolaires et de monographies qui ne les explicitent pas non plus. La question suivante s'impose : Dans quelle mesure est-il permis de dire que les systèmes d'enseignement des sciences et des techniques rendent, ou ne rendent pas, les moyens de cette traduction disponibles pour les professeurs ? 
Pour apporter des éléments de réponse à cette question, nous estimons convenable de donner de la praxéologie en question une esquisse. La feuille étant menue d'un système cartésien de repérage des points de la feuille, l'esquisse s'articule autour de la lecture de tracés sur la feuille comme sténogrammes de sériations chronologiques de points de l'axe des ordonnées quand l'axe des abscisses est l'axe des temps. A ce propos, remarquons que les premiers enseignements du repérage de points sur une feuille de papier interviennent dans les premières années de l'enseignement obligatoire et le rangement chronologique des états d'êtres vivants est une activité à laquelle on initie habituellement les enfants dès la maternelle.

En quoi la sériation des points de l'axe des ordonnées, via un tracé sur la feuille et via l'axe des abscisses comme axe des temps, est-elle une praxéologie ? Ou comment cette sériation engendre-t-elle un questionnement spécifique se présentant comme conceptualisation d'un domaine de connaissances en attente de spécification et de formalisation?

En guise d'éléments de réponse à cette question relevons des faits fondant le questionnement indiqué :

- $\quad$ Si les points sériés, selon la méthode indiquée ci-dessus, sont des positions à des instants donnés d'un point animé d'un mouvement rectiligne de trajectoire l'axe des ordonnées et si chacun des tracés considérés doit représenter la caractéristique du mouvement d'un point unique alors rejeter par principe l'ubiquité nécessite distinguer dans l'ensemble des tracés deux classes, l'une complémentaire de l'autre : les tracés qui sont caractéristiques de mouvements rectilignes d'un point et les tracés qui ne peuvent pas l'être. Cette distinction est fondamentale, en mathématique elle est le trait distinctif entre les courbes qui sont représentations graphiques de fonction numérique de la variable numérique et les autres. Un tracé peut être partitionné en un nombre de morceaux (le plus petit possible) de telle sorte que chacun de ces morceaux soit caractéristique du mouvement rectiligne d'un point. Ainsi, si les axes du tracé du chiffre huit sont parallèle aux axes du repère quelle partition peut-on opérer sur ce tracé pour obtenir le plus petit nombre de ces morceaux ? La même question se pose pour le tracé du symbole représentant l'infini. A propos de ces deux figures, pour quelle position dans l'axe des ordonnées et pour quel point mobile (caractérisé par le tracé caractéristique de son mouvement) le passage du mobile dans cette position pourrait être qualifié d'infiniment fugace et quelle position ce passage pourrait-il être qualifié de lourdement tenace?

- $\quad$ Pour certains tracés, un même point peut être la position, à des instants différents, du point mobile. Cette position peut être atteinte alors que le point est en ascension ou en descente. La question s'impose de comparer les deux arrivés à la position et les deux départs de la position : une des deux arrivées serait plus brusque, plus lente que l'autre ou les deux seraient-elles d'égale vélocité ? La même question se pose pour les deux départs.

On peut aussi faire se chevaucher les trajectoires, contenues dans l'axe des ordonnés (l'axe des abscisses étant l'axe des temps) par un choix adéquat de tracés représentant des caractéristiques de mouvements rectilignes de points. On peut ainsi organiser via ce choix le rattrapage d'un mobile par autre en assurant notamment un avantage, en temps ou en espace, au mobile à la célérité désavantagée. On peut à cette occasion introduire l'équivalence d'un avantage en temps avec un avantage en espace donnés à ce mobile, l'argument de cette équivalence est d'imposer que le rattrapage du mobile à la célérité désavantagée par l'autre mobile à la célérité avantagée se produise soit dans une position donnée soit en un instant donné.

Soumis à la catégorisation dictée par le choix de rejeter l'ubiquité hors du champ d'étude et transformés, via les questions ci-dessus, en un objet d'étude à part entière, les tracés sur une feuille de papier s'ouvrent à un champ d'interprétations et de manipulations diverses. 
Assigner à ces manipulations une motricité stylisée serait la marque d'une conceptualisation spécifiée de ce champ.

La motricité stylisée dont il s'agira ne saurait être que celle immanente à une sténographie de figement de représentations scéniques de mouvements rectilignes. Encore faudrait-il que ces dernières soient associées à de segmentations thématiques épousant la forme de sériations chronologiques de positions de points mobiles. Ceux-ci meuvent dans l'axe des ordonnées du système cartésien des coordonnées rectangulaires dont on doterait le plan. Lequel plan doit être représenté par un support d'écriture comme la feuille de papier.

Ce sont ces représentations scéniques de mouvements que l'on rejette sur ces segmentations thématiques que nous désignons ici perceptions kinesthésiques externes. Nous entendons par externes, provenant de l'extérieur du corps. D'où cette question fondamentale : Comment ériger des tracés sur une feuille de papier en une sténographie de perceptions kinesthésiques externes?

\section{B. Vers une sténographie de perceptions kinesthésiques externes}

\section{Un préalable à l'introduction, en classe, des 3 segmentations thématiques associées au triangle rectangle et à la représentation scénique du mouvement qu'elles spécifient.}

Un tracé sur une feuille de papier s'il est ouvert et ne se tourne pas sur lui-même se transforme en la caractéristique d'un mouvement rectiligne une fois la feuille dotée d'un système de repérage de ses points, un système cartésien de cordonnées rectangulaires convenablement placé.

En effet, une segmentation thématique de l'axe des ordonnées est toujours induite par le tracé et une subdivision opérée sur la projection de ce tracé sur l'axe des abscisses parallèlement à l'axe des ordonnées :

- L'axe des ordonnées étant considéré axe des temps, la segmentation en question se traduit par une sériation chronologique de points de l'axe des ordonnées ;

- Chacun des points sériés est la position, à un instant déterminé, du point doué du mouvement dont la caractéristique est le tracé en question ;

- Si cette sériation admet un dernier point, on peut convenir qu'il y a disparition du mobile immédiatement après l'atteinte de cette position.

- En changeant de repère tout en gardant la correspondance 'subdivision-sériation chronologique' le mouvement voit ses caractéristiques changer. Changer de repère équivaut à opérer un certain déplacement du tracé.

Ainsi, représentés par des tracés sur une feuille dotée d'un système de repérage, des mouvements rectilignes peuvent faire l'objet d'une représentation scénique comme interprétation de la sériation chronologique mentionnée ci-dessus.

- Amener les élèves à se faire une telle interprétation à une telle sériation ;

- les amener à donner de ce genre d'interprétations-représentations une description de plus en plus fine et de moins en moins arbitraire à mesure qu'on passe d'un niveau d'enseignement au niveau supérieur.

Telles sont, entre autres finalités, les finalités des systèmes d'enseignement des sciences et des techniques.

Pour atteindre ces finalités, il est nécessaire d'arriver dans les interprétations-représentation en question à un stade de finesse assez élevé. Arriver à cette finesse est nécessaire aussi avant 
d'aborder les trois segmentations thématiques associées au triangle rectangle et à la représentation scénique du mouvement que ces segmentations figent. Pour y arriver, il est nécessaire d'admettre que certaines questions, parmi celles que nous présentons ci-dessous, peuvent ériger des tracés muets en de sténogrammes de mouvement rectilignes. Présentons d'abord le contexte à même de motiver ces questions.

A propos de ces questions, voici un contexte pouvant les motiver : On convient que le choix d'un triangle OIJ rectangle en $\mathrm{O}$ sur une feuille de papier dote la feuille d'un système de repérage de ses points, système dit de cordonnées rectangulaires dont les couples $(\mathrm{O} ; \mathrm{I})$ et $(\mathrm{O} ; \mathrm{J})$ sont les bipoints unitaires. Si on convient que l'axe $(\mathrm{O} ; \mathrm{I})$ est axe des temps et que l'axe $(\mathrm{O} ; \mathrm{J})$ est axe des positions d'un point mobile $\mathrm{M}$. Un tracé sur la feuille peut donner lieu, via un choix pertinent du repère $(\mathrm{O} ; \mathrm{I} ; \mathrm{J})$ (donc du triangle $\mathrm{OIJ})$, à un sténogramme d'un mouvement rectiligne. Souvent ce choix du triangle OIJ est contraint par la nature du tracé et par quelques propriétés choisies a priori pour le mouvement pour lequel on veut que ce tracé soit, à côté du triangle OIJ choisi, un des éléments constitutifs du sténogramme. Rappelons que ce choix peut être réalisé en fixant OIJ et en déplaçant convenablement le tracé à la faveur d'une rotation autour d'un point, d'une translation dans une direction donnée, une symétrie par rapport une droite ou d'une combinaison de ces trois transformations. Dans le paragraphe suivant, nous présentons des exemples de ces questions.

\section{Questions introductives d'une sténographie de mouvements}

- Considérons le tracé suivant constitué de deux segments :

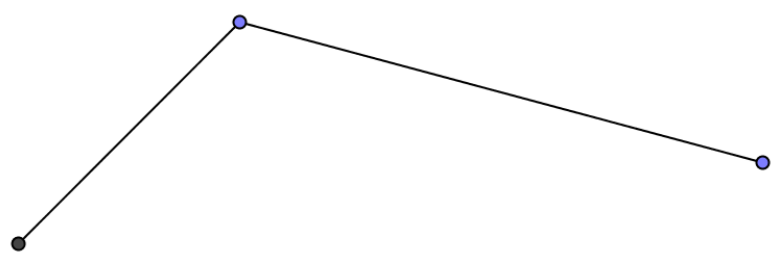

On choisit le triangle OIJ de telle sorte que «l'horizontale» soit la direction de (OI), la verticale celle de $(\mathrm{OJ}), \mathrm{J}$ au-dessus de $\mathrm{O}$ et $\mathrm{I}$ à droite de $\mathrm{O}$. On convient que les départs s'effectuent à l'instant 0 , et que le point de départ est $\mathrm{O}$ et quand ce n'est pas le cas, le mobile commence par s'approcher de $\mathrm{O}$ jusqu'à l'atteindre avant de continuer son mouvement en 
s'en éloignant dans un sens ou dans l'autre. Dans ces conditions comment placeriez-vous ce tracé pour obtenir, via la sériation chronologique introduite ci-dessus, les représentations des mouvements rectilignes répondant aux caractéristiques suivantes :

1) A l'instant 0 , le mobile fait son apparition au-dessus du point $\mathrm{O}$ et commence à s'approcher de $\mathrm{O}$ jusqu'à l'atteindre. Immédiatement après, il s'en éloigne :

a. soit en rebroussant chemin, avant de disparaître un certain temps après ;

b. soit en continuant au-dessous de $\mathrm{O}$, tout en changeant de régime, avant de disparaître un certain temps après.

Il est question, à ce propos, d'envisager aussi, par un passage à la limite que vous décrirez et représenterez, les deux cas suivants :

i. à l'instant 0 , le mobile 'saute' sur $\mathrm{O}$ à partir d'une position située audessus de $\mathrm{O}$ et commence par s'éloigner de $\mathrm{O}$ vers le bas avant de disparaître un certain temps après ;

ii. à l'instant 0 , le mobile apparaît sur $\mathrm{O}$, s'y immobilise pendant quelque temps, avant de commencer à s'en éloigner vers le haut et disparaître un certain temps après.

2) A l'instant 0 , le mobile part de $\mathrm{O}$, s'en éloigne, vers le haut, pendant un certain temps. La disparition du mobile survient ensuite. Elle survient chaque fois dans l'une ou l'autre des 4 types de positions suivantes :

a. à son arrivée à une position située entre $\mathrm{O}$ et une position au niveau de laquelle il avait commencé à rebrousser chemin ;

b. après qu'il s'est immobilisé dans une position au-dessus de $\mathrm{O}$ pendant un certain temps ;

c. à son arrivée à une position située au-dessus de $\mathrm{O}$ et après que le mouvement ait changé, entre temps, de régime ;

d. à son retour à la position $\mathrm{O}$, après que le mouvement ait changé de régime une fois.

e. à son arrivée à une position située au-dessous de $\mathrm{O}$ et après que le mouvement ait changé de régime une fois.

Envisager, par un passage à la limite que vous décrirez et représenterez, les deux cas suivants :

i. à l'instant 0 , le mobile 'saute', à partir de $\mathrm{O}$, sur une position située au-dessus de $\mathrm{O}$ et continue à s'éloigner de $\mathrm{O}$ vers le haut avant de disparaître un certain temps après ;

ii. à l'instant 0 , le mobile fait son apparition en $\mathrm{O}$, s'immobilise dans cette position pendant un certain temps avant de la quitter et disparaître après s'en être éloigné pendant un certain temps.

\section{Sténogrammes de mouvements dont le tracé ci-dessus, à un déplacement près, est élément constitutif}

- Sténogramme du mouvement décrit en 1) a.

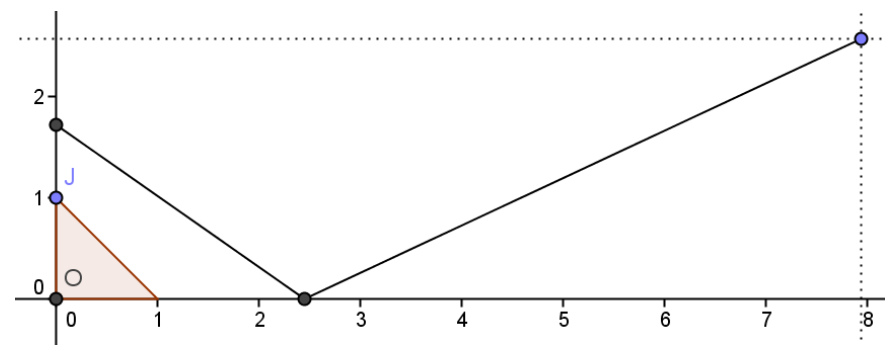


- Sténogramme du mouvement décrit en 1) b.

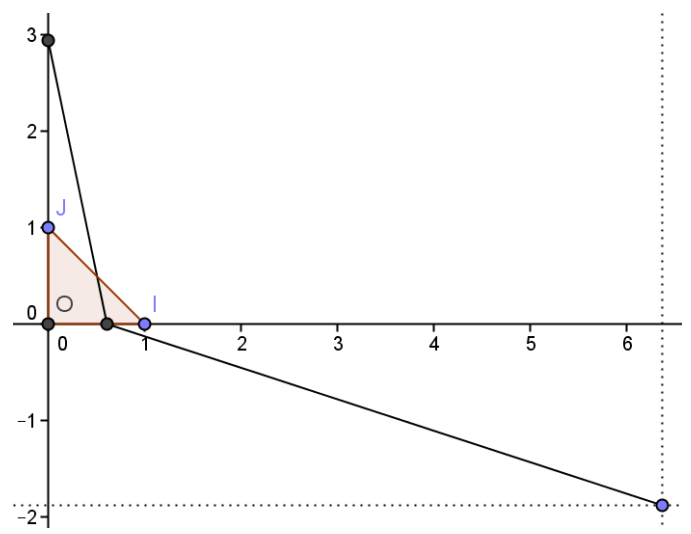

- Sténogramme du mouvement décrit en 1) i.

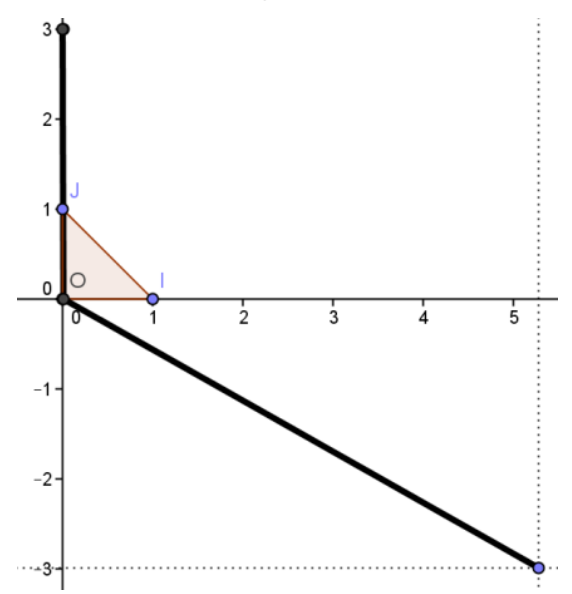

- Sténogramme du mouvement décrit en 1) ii. 


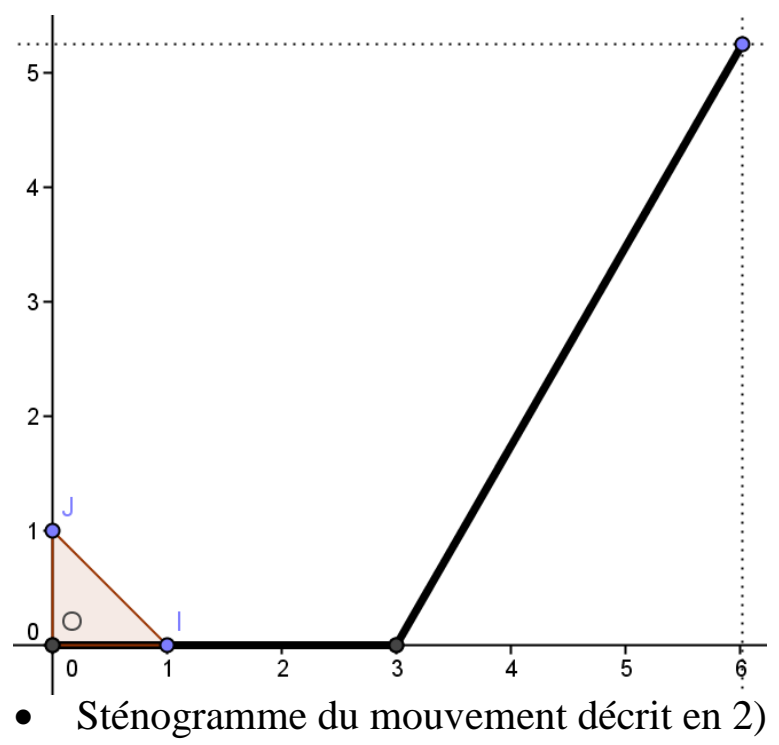

- Sténogramme du mouvement décrit en 2) a.

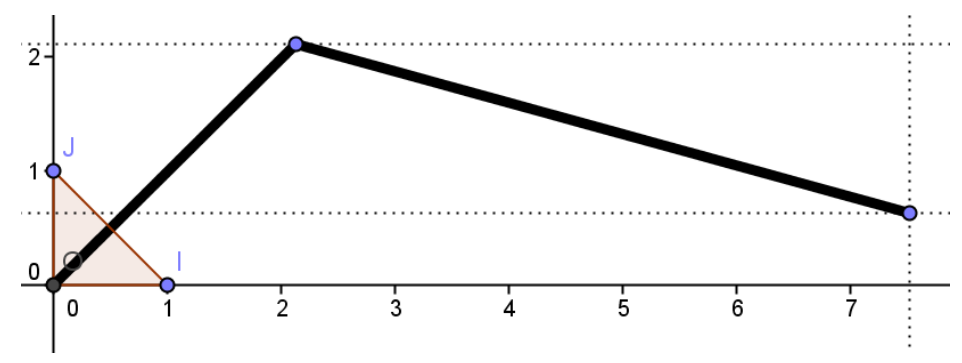

- Sténogramme du mouvement décrit en 2) b.

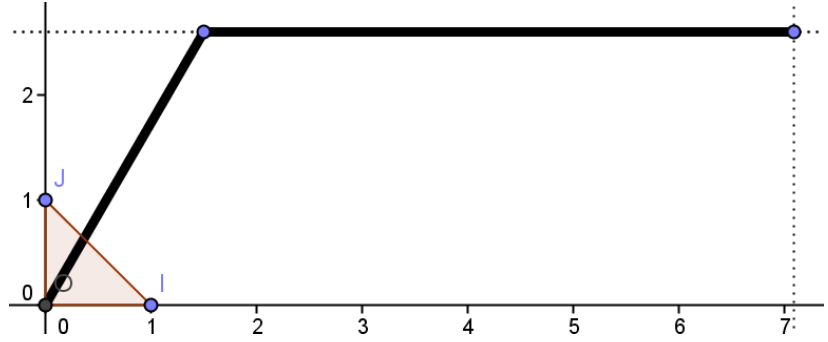

- Sténogramme du mouvement décrit en 2) c.

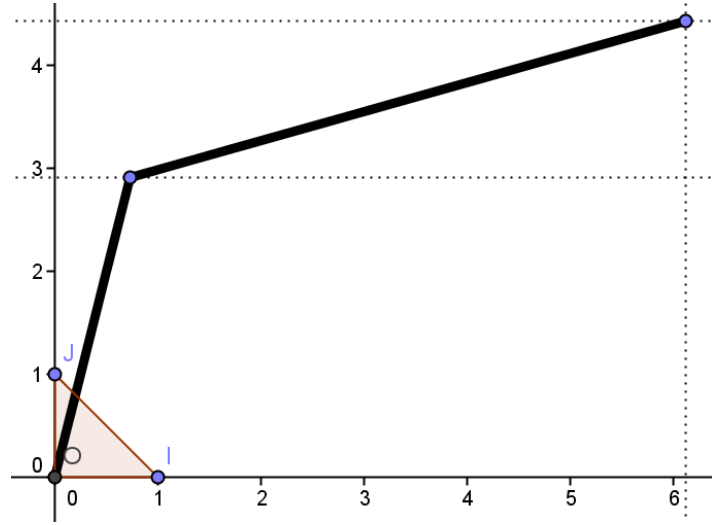


- Sténogramme du mouvement décrit en 2) d.

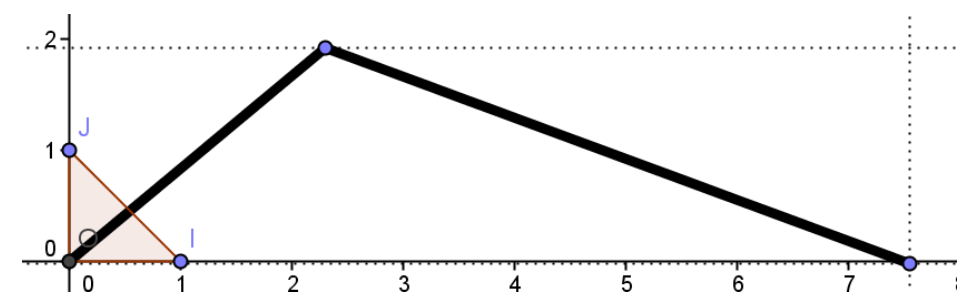

- Sténogramme du mouvement décrit en 2) e.

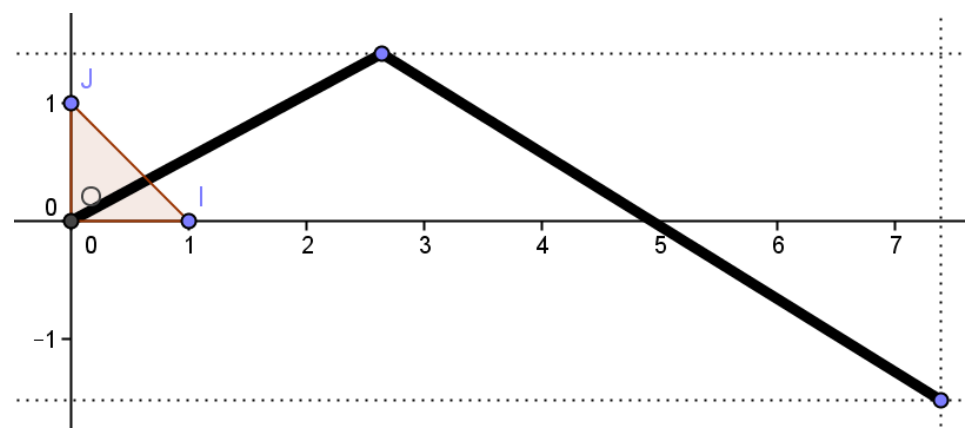

- Sténogramme du mouvement décrit en 2) i.

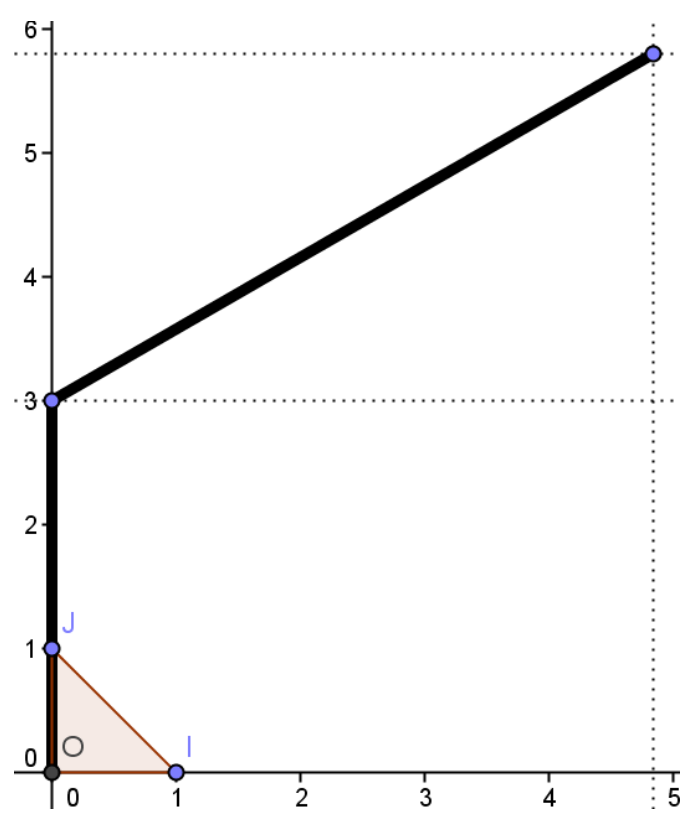


- Sténogramme du mouvement décrit dans 2) ii.

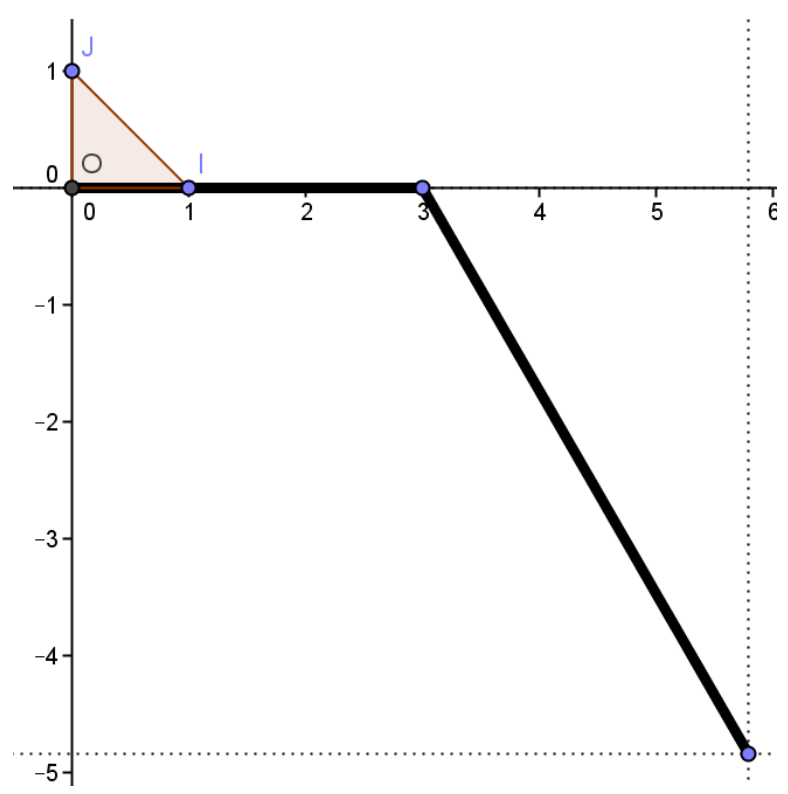

\section{A.C. Des 3 segmentations thématiques associées au triangle rectangle aux sténogrammes de perceptions kinesthésiques externes}

Les sténogrammes ci-dessus sont de nature à jouer un rôle fondamental quant aux réponses que l'élève doit apporter aux questions de la fin du paragraphe déroulé plus haut et intitulé «Questions fondant la représentation scénique interprétatrice des trois segmentations thématiques du triangle rectangle ».

En effet, il est tout à fait convenable de chercher les sténogrammes constitutifs des réponses aux questions en arrondissant des parties anguleuses de sténogrammes introduits dans le paragraphe ci-dessus. Une dialectique entre cette action de recourbement et la segmentation de second degré issue des trois segmentations thématiques associées au triangle rectangle finirait par établir les sténogrammes de perceptions kinesthésiques externes.

On peut retrouver ces sténogrammes par un calcul sans avoir à introduire les sténogrammes intermédiaires ci-dessus ni à arrondir la partie anguleuse. Mais ce calcul est un outil trop puissant qui synthétise et cache une trop grande complexité. Donner du sens à ce calcul consiste à former une idée assez précise de la complexité cachée. La dialectique que nous 
venons de suggérer participe en fait d'une technique de déploiement de cette complexité donnant au calcul tout son sens.

- Sténogramme du mouvement décrit en 2) b.

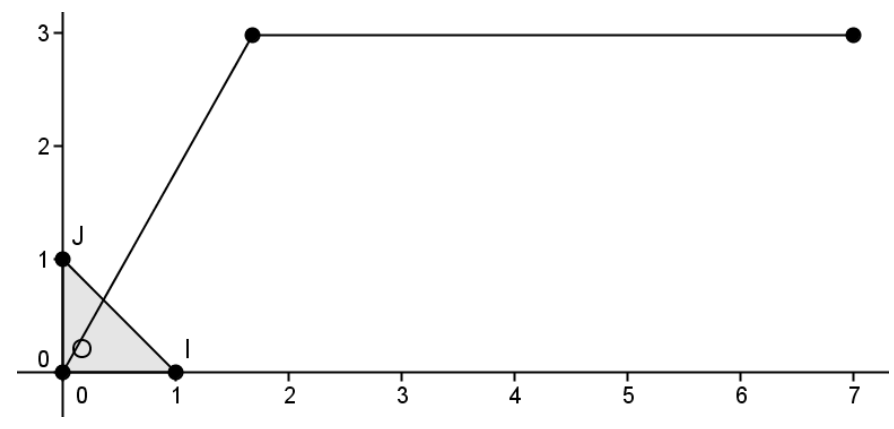

- Traduction surfacique du sténogramme

On considère :

$\bullet$ un rectangle $\mathrm{OABC}$, avec

$\mathrm{A}$, un point de $(\mathrm{OJ}), \mathrm{C}$ un point de $(\mathrm{OI})$;

-0 un point $\mathrm{D}$ de $(\mathrm{OI})$ d'abscisse d, avec C entre $\mathrm{O}$ et $\mathrm{D}$;

- o la fonction f, définie par : à tout réel $\mathrm{t}, 0 \leq \mathrm{t} \leq \mathrm{d}$, fait correspondre l'aire de la surface de la partie du polygone OABCD comprise entre (OA) et la

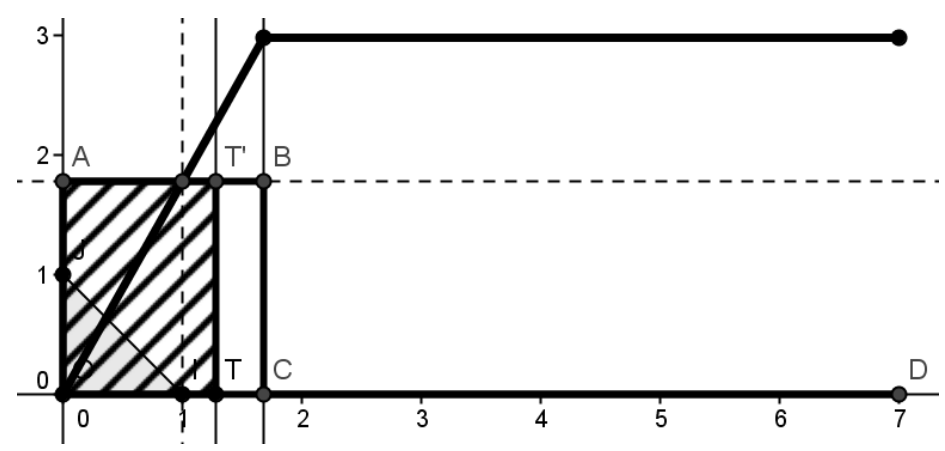
perpendiculaire en $\mathrm{T}$ à (OI), T, point de (OI) d'abscisse $t$;

la courbe $\mathrm{C}_{\mathrm{f}}$ et le sténogramme sont confondus. 


\section{Un procédé pour un recourbement du coin anguleux du sténogramme}

Le recourbement du point

anguleux s'effectue en

remplaçant :

- Dans le polygone OABCD : le rectangle OABC par le triangle OA'C, avec A', le symétrique de $\mathrm{O}$ par rapport à $\mathrm{A}$;

- Dans la définition de $\mathrm{f}$ : « la surface de la partie du polygone $\mathrm{OABCD} »$ par «la surface de la partie du polygone $\mathrm{OA}$ 'CD ».

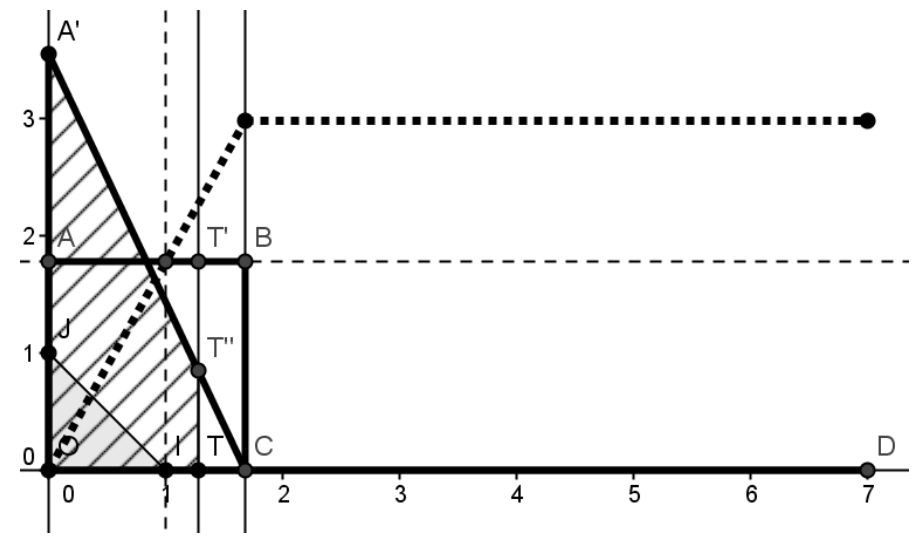

Ce procédé vise garder la même position d'arrêt et le même instant d'arrivée à cette position tout en organisant l'arrêt par un freinage constant qui fait coïncider l'annulation de la vitesse avec l'instant d'arrivée à la position.

D'où ce transformé du sténogramme (ou nouvelle courbe $\mathbf{C}_{\mathrm{f}}$ )

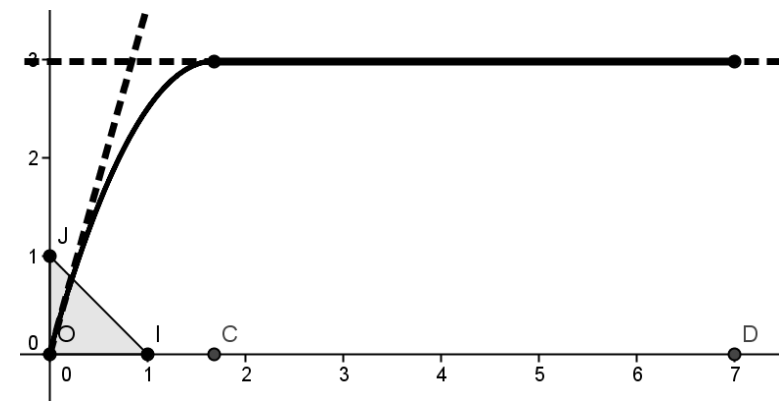




\section{Troisième partie}

Confrontation de la praxéologie et de la sténographie cognitive présentées à la contingence: Une représentation scénique erronée chez la quasi-totalité des étudiants marocains?

Considérons les deux questions suivantes:

- Est-il nécessaire, pour les systèmes d'enseignement des sciences et des techniques, de faire de ces 3 segmentations thématiques du triangle rectangle, et de la représentation scénique kinesthésique qui leur sont associée, des objets d'enseignement à part entière (i.e., des connaissances officiellement exigible de tout bachelier?)

- Existe-t-il des arguments assez forts en faveur d'une réponse par l'affirmative à cette question?

Un de ces arguments envisage des situations-problèmes dont la solution consiste à considérer l'axe des abscisses, axe des temps, l'axe des ordonnées, trajectoire de points mobiles, les courbes représentatives de fonctions numériques de la variable réelle, des procédés pour localiser, pour des instants déterminées, les positions de tels points (le plan étant doté d'un repère orthonormé). Il est un argument fort le fait qu'au sein d'un de ces systèmes, une proportion importante des bacheliers des sections à forte teneur mathématique s'avère incapable de solutionner de telles situations. Il est possible que ce soit le cas pour le système marocain, au vu des résultats du test, ci-dessous, passé à des hordes d'étudiants.

\section{Énoncé}

Ce schéma est censé représenter le parcours d'un automobiliste qui, après s'être arrêté et garé sa voiture dans le bas-côté d'une route (une cachette), il a redémarré sa voiture et repris sa route. Après avoir atteint sa vitesse de croisière, il a observé devant lui, à quelque centaines de mètres, un obstacle (par exemple, un policier qui lui fait

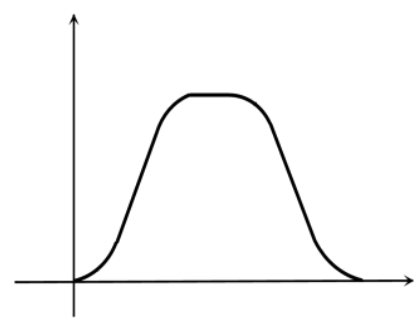
signe de s'arrêter). Il ralentit, pour enfin s'arrêter au niveau de cet obstacle. Après un arrêt, il fit demi-tour pour rejoindre sa cachette.

\section{Questions :}

- Est-ce que le schéma représente effectivement ce qu'il est censé représenter?

- Sinon pourquoi ? Si oui où placeriez-vous l'obstacle (mettre une croix dans le schéma sur la position choisie et justifiez votre réponse)? 
Résultats obtenus dans différents établissements :

\begin{tabular}{|c|c|c|c|c|}
\hline$\underline{\text { Filière }}$ & $\underline{\text { Niveau }}$ & $\underline{\underline{E} \text { tablissement }}$ & $\underline{\mathbf{N}^{\text {bre }} \text { Étudiants }}$ & $\underline{\underline{\mathbf{N}^{\text {bre }}} \text { bonnes réponses }}$ \\
\hline$\underline{\text { BTS/P.C. }}$ & $\underline{\mathrm{Bac}+2}$ & $\underline{\text { ENSET /Casa }}$ & $\underline{18}$ & $\underline{0(0 \%)}$ \\
\hline$\underline{\text { BTS/ E.I. }}$ & $\underline{\mathrm{Bac}+2}$ & $\underline{\text { ENSET / Casa }}$ & $\underline{23}$ & $\underline{0(0 \%)}$ \\
\hline$\underline{\text { CAPES/ Math. }}$ & $\underline{\mathrm{Bac}+5}$ & $\underline{\text { ENSET / Casa }}$ & $\underline{28}$ & $\underline{0(0 \%)}$ \\
\hline$\underline{\text { CAPES/P.C. }}$ & $\underline{\mathrm{Bac}+5}$ & $\underline{\text { ENSET/Rabat }}$ & $\underline{27}$ & $\underline{0(0 \%)}$ \\
\hline$\underline{\text { DEUG/Sciences }}$ & $\underline{\mathrm{Bac}+3}$ & $\underline{\text { CPR/Safi }}$ & $\underline{22}$ & $\underline{1(4,5 \%)}$ \\
\hline$\underline{\text { Total }}$ & & & $\underline{118}$ & $\underline{1(0,84 \%)}$ \\
\hline
\end{tabular}

\section{$\underline{\text { Conclusion }}$}

Nous concluons en insistant sur le fait que l'école de demain sera celle des variables spatiotemporelles, comme variables de commande entre les mains du professeur et nous recommandons aux professeurs de commencer, dès maintenant, à s'habituer à articuler leurs discours autour d'une mobilisation optimale de ces variables et ce, en s'inspirant notamment des sténogrammes présentés ci-dessus.

Pris avec leurs renvois à la fois à l'étude qui vient d'en être faite et à la nature du test passé aux étudiants et les résultats enregistrés, les rapports, institutionnel, personnel et culturel au triangle rectangle constituent un argument fort en faveur de cette recommandation. En effet, le triangle rectangle ne saura rester trop longtemps encore une forme statique. L'acception kinesthésique que nous venons de dérouler à propos de cette forme géométrique deviendra un objet d'enseignement à part entière : les systèmes d'enseignement des sciences et des techniques seront, à terme, contraints de considérer l'accélération, prise avec son renvoi à une force ayant un effet accélérateur ou un effet frein sur le mouvement ascendant ou descendant d'un point mobile, la vitesse instantanée, le procédé de localisation de ce point et la modélisation, qui en découle, du mouvement du projectile, un système d'objets de savoir à institutionnaliser comme tel dès le collège, voire dès le primaire, notamment dans le cadre des activités de sériation chronologique. 


\section{REFERENCES BIBLIOGRAPHIQUES}

Aazim, J. A. «Les déterminants pragmatiques de la professionnalisation du métierd'enseignant dans une approche cognitive - Cas de l'Enseignement des Sciences et des Techniques ». Thèse nationale, faculté des sciences Ben M'sik, Université Hassan II Mohammedia/Casablanca. 2012.

Bachimont, B. «Le contrôle dans les systèmes à base de connaissances, contribution à l'épistémologie de l'intelligence artificielle ». Paris : Hermès. 1999.

Bahra M., Bouanani H.,. « La conception sténographique et la conception kinesthésique de la fonction numérique de la variable réelle, Les nouvelles technologies pour l'enseignement des mathématiques » http://revue.sesamath.net/spip.php?article431. 2012

Benveniste, E. « Problèmes de linguistique générale ». Paris, Gallimard.1974.

Boucheron, S. «Théorie de l'apprentissage, de l'approche formelle aux enjeux cognitifs ». Paris : Hermès. 1992.

Bouanani, H. «Des configurations de points aux ensembles de points, problèmes de didactique de la nécessitation de l'abstraction en mathématiques ». Thèse nationale, faculté des sciences Ben M'sik, Université Hassan II - Mohammedia/Casablanca. 2012

Brousseau, G. «La théorie des situations didactiques ». Textes rassemblés et préparés par N. Balacheff, M.Cooper, R. Sutherland \& V. Warfield). Grenoble : La Pensée Sauvage. 1998

Brousseau, G.. «Les obstacles épistémologiques, problèmes et ingénierie didactique »; in G. Brousseau, Théorie des situations didactiques (pp. 115-160). Grenoble : La Pensée Sauvage. 1998

Brousseau, G. «Glossaire de quelques concepts de la théorie des situations didactiques en mathématiques ». En ligne http://pagesperso-orange.fr/daest/guy brousseau/textes/Glossaire_Brousseau.pdf, consulté le 23/01/2009. 2003.

Brousseau, G. «L'ingénierie des situations mathématiques ». En ligne http://guybrousseau.com/tag/ingenierie-didactique/, consulté le : 10/01/2011. 2010.

Desanti J-T « La philosophie silencieuse ». Paris, Seuil. 1975.

Dessalles, J-L. « Aux origines du langage ». Paris, Hermès Science 2000.

El Abbassi, F. «Caractérisation de la structure fonctionnelle du Discours Enseignant. Contribution à la catégorisation de l'intersubjectivité en enseignement des sciences et des techniques ». Thèse nationale, faculté des sciences Ben M'sik, Université Hassan II Mohammedia/Casablanca. 2012.

Frege, G., Imbert, C. «Ecrits logiques et philosophiques ». Paris. : Ed. du seuil. 1971.

Granger, G-G. « Philosophie, langage, science ». Editions : Les Ulis, EDP sciences. 2003.

Hofstadter, D. «Gödel, Escher, Bach. Les brins d'une guirlande éternelle ». Paris: Dunod. 2000.

Husserl, E. «Recherches logiques : Recherches pour la phénoménologie et la théorie de la connaissance ». (tome 2) (Hubert E., Arion L. Kelkel \& Schérer R. trad.). Paris: Presses universitaires de France. 1969. 
Husserl, E. «Recherches logiques, : Eléments d'une élucidation phénoménologique de la connaissance ». (tome 3) (Hubert E., Arion L. Kelkel \& Schérer R. trad.). Paris: Presses universitaires de France. 1974.

Johsua, s. \& Dupin, J. J. «Introduction à la didactique des sciences et des mathématique ». Paris : Presses Universitaires de France. 1993.

Kotarbiński «Les origines de la praxéologie », Académie Polonaise des Sciences, fascicule 58, PWN., Warszawa, 1965. (PWN, Warszawa, , p. 18), 1962.

Kuzniak, A. (S.d.). «La théorie des situations didactiques de Brousseau ». En ligne http://irem.u-strasbg.fr/php/articles/110_Kuzniak.pdf, consulté le 24 janvier 2009

Ladrière, P., Pharo, P. \& Queré, L. «La théorie de l'action, Le sujet pratique en débat ». Paris : CNRS. 1993.

Le Moigne, J. L., Morin, E. «Intelligence de la complexité, Epistémologie et pragmatique ». France : Ed. de l'Aube. 2007.

Milner, J. C. «Introduction à une science du langage ». Paris : Ed. Seuil. 1989.

Montréal: Éditions Logiques.

Najoua, A. "Etablissement et validation d'un schéma de structuration des contenus d'enseignement à teneur praxéologique - Cas de l'enseignement des sciences et des techniques ». Thèse nationale, faculté des sciences Ben M'sik, Université Hassan II Mohammedia/Casablanca. 2012.

Rastier, F. «L'action et le sens pour une sémiotique des cultures. Journal des anthropologues », $n^{\circ} .85-86, \quad p p$. 183-219. En ligne http: www.revuetexto.net/Inedits/Rastier/Rastier_Action.html. Consulté le 22 mars 2010. 2001.

Stichweh, R. «Etudes sur la genèse du système scientifique moderne ». Traduction de F. Blaise. Lille : Presses Universitaires. 1991.

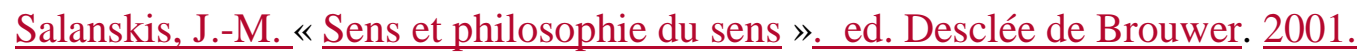

Sarfati, G-E. « Les éléments d'analyse du discours ». Paris, Armand colin. 2003

Tutescu, M. «L'Argumentation, introduction à l'étude du discours ». Bucaresti, Editura universitatii din Bucaresti. 2005. 